\title{
Agriculture et risques de pollution diffuse par les produits phytosanitaires
}

\author{
Les voies de la prévention et les apports de l'expérience Ferti-Mieux ${ }^{1}$
}

\section{Agriculture and diffuse pollution risks generated by plant protection products}

\author{
par Michel Sebillotte \\ Professeur d'Agronomie \\ Directeur scientifique à I'INRA \\ Président du comité scientifique de Ferti-Mieux \\ Membre du bureau du CORPEN
}

The issue of water contamination with plant protection products is one which is being raised ever more frequently. But in fact a lot of useful experience has already been gained in avoiding the risk of nitrate pollution thanks to FertiMieux operations which are based on an obligation to use cultivation techniques which reduce the risks of pollution as much as possible. The article sets out to demonstrate how this experience can also be applied to the case of plant protection products. In order to do this, we begin by examining the differences between nitrogen and plant protection products, especially in terms of the responsibilities of farmers and their consultants. Then we deal with prevention methods, training the farmers, and the concepts of crop systems and land management. In the conclusion we discuss joint action measures aimed at risk prevention.

\section{INTRODUCTION}

La question de la pollution des eaux par les produits phytosanitaires est de plus en plus souvent soulevée, entre autres au sein des opérations Ferti-Mieux 2. Ces opérations de prévention, basées sur le volontariat, visent à réduire les risques de pollution diffuse par les nitrates avec, comme principe d'action, une " obligation de moyens ", celle de modifier les pratiques pour les rendre plus respectueuses de l'environnement. Ne pourrait-on, cela serait si simple, reproduire ce modèle pour les produits phytosanitaires ? Nous verrons au cours de cet exposé comment, malgré des différences de fond entre l'azote et les produits phytosanitaires, l'expérience "Ferti-Mieux" peut fournir un cadre de réflexion adéquat pour améliorer les pratiques de lutte contre les ennemis des cultures.

\footnotetext{
1 Texte de la Conférence prononcée au Congrès AGHTM - Association Générale des Hygiénistes et Techniciens Municipaux, Dijon 11-14 mai 1998.

2 Le siège de l'opération nationale dont l'initiative revient à la profession agricole et au ministère de l'Agriculture, se trouve à l'Association Nationale pour le Développement Agricole à Paris. Les agriculteurs qui adhèrent aux différentes opérations Ferti-Mieux représentent une surface cultivée de plus d'un million d'hectares.
}

\section{II — LES DIFFÉRENCES ENTRE L'AZOTE ET LES PRODUITS PHYTOSANITAIRES}

Bâtir une opération pour les produits phytosanitaires en " recopiant" les règles de Ferti-Mieux négligerait la différence radicale qui existe entre l'azote et ces produits, et risquerait donc de conduire à l'échec.

En effet, l'azote fait partie des cycles biogéochimiques naturels, il est présent dans le milieu sans aucune intervention humaine, ce qui n'est pas le cas des produits phytosanitaires. L'azote minéral, celui qui pollue les eaux lorsque sa teneur dépasse le seuil de $50 \mathrm{mg} / \mathrm{l}$, est produit spontanément sous l'action des micro-organismes du sol, à partir des matières organiques qui s'y trouvent, elles-mêmes originaires, pour l'essentiel, des végétaux, cultivés ou non. Par ailleurs, l'azote fait partie de la constitution des plantes cultivées ; elles ont besoin d'azote car il joue un rôle essentiel pour leur croissance et leur reproduction. Cela explique les pratiques de fertilisation des agriculteurs. En effet, lorsque les fournitures du milieu sont insuffisantes vis-à-vis des besoins des cultures pour atteindre les objectifs de production, il faut les compléter par des apports d'engrais. En utilisant les lois qui régissent la dynamique du cycle de l'azote, mises en évidence par la recherche, il est possible d'estimer les fournitures d'azote sous forme minérale et de les confronter aux besoins calculés des cultures et donc de 
déterminer les quantités à apporter, à partir des amendements organiques et/ou des engrais. C'est la méthode du bilan prévisionnel pour raisonner la fertilisation azotée.

Dans la pratique les choses ne sont pas si simples. En effet, le climat reste imprévisible ! Or, il a un rôle considérable sur la dynamique de l'azote (minéralisation et pertes en profondeur) et sur le fonctionnement des plantes cultivées (et de leurs " ennemis ") et donc sur leur potentiel de production. Néanmoins, les données scientifiques et techniques actuelles permettent de réduire considérablement les risques en fournissant des outils de raisonnement pour maintenir au mieux un équilibre entre les besoins des cultures et les fournitures par le milieu et les apports, règle de base de la Directive "Nitrates".

Par contre les produits phytosanitaires issus de l'industrie ne se trouvent pas spontanément dans la composition des végétaux (ni des animaux), et, dans leur grande majorité, ce ne sont pas des substances naturelles ${ }^{3}$. C'est une première différence essentielle avec l'azote : les plantes n'ont pas besoin pour croître de ces molécules. On les utilise pour lutter contre les " ennemis" des cultures, mauvaises herbes, champignons, insectes, etc. La présence de ces " ennemis " nuit, en effet, à la production des cultures s'ils sont trop abondants ou interviennent à un moment défavorable. L'usage des produits phytosanitaires ne vise donc pas à couvrir des besoins de la culture ${ }^{4}$ mais à maintenir un niveau de production donné en contrôlant des facteurs limitants. La notion d'équilibre entre des besoins et des apports n'existe pas ici ; c'est la deuxième différence essentielle par rapport à l'azote !

\section{III — LES PRODUITS PHYTOSANITAIRES : DES PRODUITS INDUSTRIELS DONT L'USAGE EST TRÈS CODIFIÉ}

A cause de ces deux différences et des risques de toxicité pour les êtres vivants, la mise en marché de produits phytosanitaires par l'industrie est soumise à des règles très strictes d'homologation qui doivent garantir l'absence de risques pour la santé (homme et animaux), les eaux et l'environnement lorsque ces produits sont " convenablement utilisés, eu égard aux principes des bonnes pratiques ${ }^{5}$ phytosanitaires et de la lutte intégrée contre les ennemis des végétaux ".

C'est par la recherche que l'on détermine les risques, puisque précisément on ne peut s'appuyer sur le caractère naturel de ces substances ni sur des règles issues d'une longue tradition d'emploi. Tout une série d'obligations pour les industriels ont été codifiées par la directive de 1991 et ont été actualisées et précisées dans une seconde directive de $1997^{6}$. Ainsi, c'est aux industriels que revient en particulier la tâche de préciser " les conditions agricoles, phytosanitaires et environnementales spécifiques dans lesquelles le produit peut être utilisé ou doit, au contraire, être exclu " et " les instructions d'emploi et la dose à appliquer pour chaque usage autorisé ", indications qui doivent figurer sur " tout emballage (...) de manière lisible et indélébile " 7 . C'est ensuite aux Etats membres de contrôler que les produits phytosanitaires sont utilisés de manière à respecter les conditions édictées lors de l'homologation et qui figurent donc sur les étiquettes et les emballages.

Les textes des directives définissent ainsi les responsabilités des uns et des autres, responsabilités qui sont individuelles. Celles des agriculteurs et de leurs conseillers sont, d'abord et avant tout, de respecter les indications d'usage : date, doses et conditions agricoles d'emploi. Si tel est le cas, leur responsabilité quant à l'environnement et à la pollution diffuse n'est pas engagée. Ils ont respecté les règles qui s'imposent à eux et donc, contrairement à ce qui se passe pour la fertilisation azotée, ce ne sont ni les agriculteurs ni leurs conseillers directs qui élaborent les bases de raisonnement fixant les doses et les stades d'application ; par contre, pour décider de l'application d'un traitement ils font intervenir soit une estimation d'un risque soit un diagnostic " d'attaque " et les états du milieu 8 .

\section{IV — LA RESPONSABILITÉ DES AGRICUL- TEURS ET DE LEURS CONSEILLERS EST CEPENDANT ENGAGÉE}

Il faut, cependant, ouvrir le débat car :

- d'une part, dans le cas de pollutions, même si les agriculteurs et leurs conseillers n'étaient pas responsables, ils apparaîtraient, aux yeux des consommateurs et des citoyens, comme responsables et solidaires de fait des industriels. Leurs pratiques seraient donc mises en accusation et les agriculteurs supporteraient ainsi le risque d'un rejet de leurs produits par les consommateurs ${ }^{9}$ ou d'une condamnation morale par la société. Cela conduit donc les agriculteurs et leurs conseillers à s'interroger sur des améliorations de leurs pratiques pour diminuer les risques pouvant persister même s'il y a respect des normes d'emploi 10,11 .

- d'autre part, parce que le texte de la directive parle de " bonnes pratiques phytosanitaires et de la lutte intégrée contre les ennemis des végétaux " et précise que la lutte intégrée est " l'application rationnelle d'une combinaison de mesures biologiques, biotechnologiques, chimiques, physiques, culturales ou intéressant la sélection des végétaux dans laquelle l'emploi de produits chimiques phytopharmaceutiques est limité au strict nécessaire pour maintenir la
${ }^{3}$ Quelques produits proviennent de plantes, par exemple du pyrèthre.

${ }^{4} \mathrm{La}$ Directive 91/414/CEE dit : " considérant que l'utilisation des produits phytopharmaceutiques constitue l'un des moyens les plus importants pour protéger les végétaux et produits végétaux et pour améliorer la production de l'agriculture "

5 Directive du Conseil du 15 juillet 1991 concernant la mise sur le marché des produits phytopharmaceutiques (91/414/CEE)

6 Directive 97/57/CE du Conseil du 22 septembre 1997, établissant l'annexe VI de la directive 91/414/CEE.

7 Article 16, Directive 91/414/CEE

8 II leur arrive aussi de réduire les doses ou de procéder à des mélanges qui ne sont pas forcément recommandés et dans ce cas leur responsabilité est engagée.
${ }^{9}$ Auxquels il convient d'ajouter des industriels de la transformation qui sont de plus en plus exigeants sur ce plan.

10 Cette préoccupation des agriculteurs se retrouve dans les réflexions actuelles sur la "traçabilité ". En effet, devant les exigences croissantes des consommateurs, de certains industriels, les agriculteurs souhaitent pouvoir apporter la preuve qu'ils n'ont pas utilisé telle ou telle substance, qu'ils n'ont pas épandu de boues de station d'épuration, qu'ils ont respecté des conditions d'emploi ...

11 On peut trouver une expression de cette solidarité de fait, dans la création du " Comité de Liaison Eaux-produits antiparasitaires ", instance de liaison entre les ministères chargés de la Santé, de l'Environnement et de l'Agriculture, les commissions consultatives compétentes dans le domaine des produits phytosanitaires et le CORPEN. Cette instance définit, en particulier. des listes de substances devant être prioritairement suivies dans les eaux. 
présence des organismes nuisibles en dessous d'un seuil à partir duquel apparaissent des dommages ou une perte économiquement inacceptable $" 12$.

Il revient donc à la recherche agronomique et aux organismes intervenant dans le développement agricole de contribuer, aux côtés de la recherche-développement des industries, à mettre au point ces “ bonnes pratiques ". Les questions à traiter concernent, entre autres, les domaines des itinéraires techniques (la manière dont on cultive) et des systèmes de culture (la manière dont les cultures se suivent sur chaque parcelle et dont chacune est cultivée). Mais ces recherches pour mettre au point les " bonnes pratiques " correspondent à des actions à la parcelle. Or, si elles sont bien dans l'esprit de la directive elles peuvent être insuffisantes car, plus globalement et de manière probablement plus essentielle, les risques de pollution ne résultent pas que des pratiques individuelles mais aussi de leurs combinaisons dans l'espace. La qualité de l'eau est, pour partie, liée à la gestion de l'espace, ce qui impose à la recherche de se préoccuper aussi des effets de la localisation sur le territoire des cultures et des pratiques sur les risques de pollution par les produits phytosanitaires.

\section{V — LES VOIES DE LA PRÉVENTION}

Comme la définition de la lutte intégrée le laissait déjà prévoir, plusieurs voies doivent être empruntées simultanément pour assurer une véritable prévention. J'insisterai sur quelques points, de manière non exhaustive faute de temps : la formation et l'information des agriculteurs, la conception des itinéraires techniques et des systèmes de cultures, l'amélioration variétale, l'aménagement de l'espace cultivé.

\subsection{La formation et I'information des agriculteurs et de leurs conseillers. Les améliorations dans l'usage des produits phytosanitaires}

On peut se demander pourquoi évoquer la formation alors que tout doit être écrit sur les étiquettes. Mais se poser cette question serait oublier bien des aspects.

Le premier concerne les habitudes et leurs origines. Lorsque les règlements sur la sécurité du travail étaient moins exigeants, peu de personnes trouvaient à redire sur le fait que des agriculteurs s'exposent sans précaution particulière ! Ainsi des habitudes se sont prises qui peuvent aujourd'hui conduire certains à ne pas respecter les normes d'emploi, tout au moins à manquer de vigilance. Et lorsqu'une personne ne perçoit pas, ou mal, les risques qu'elle encourt pour elle-même, pourquoi les percevrait-elle mieux pour l'environnement ; la question mérite d'être posée. Il y a ainsi des bases objectives et sociologiques aux comportements ${ }^{13}$, renforcées par le caractère répétitif des tâches qui favorise un relâchement de la vigilance. Comme dans toute activité professionnelle, il y a donc une nécessité de formation qui doit commencer par les opérations qui se passent à la ferme ${ }^{14}$, probablement celles présentant le plus de risques, tout au moins pour la santé des opérateurs ; mais les pollutions ponctuelles ne font pas partie de mon exposé.

Le deuxième aspect que je voudrais souligner concerne la liaison entre les risques et l'organisation du travail. Prenons un exemple simple, celui du rinçage des appareils d'épandage. Avec de nombreuses machines de pulvérisation en service actuellement, cette opération de nettoyage sera, selon la position du point d'eau, plus ou moins contraignante et un agriculteur peut se trouver dans une situation où le respect des règles est très coûteux en temps. On doit donc se réjouir que les épandeurs les plus modernes possèdent une réserve d'eau pour pouvoir procéder à un rinçage au champ ${ }^{15}$. Viendra le temps où les appareils posséderont aussi un dispositif de rinçage des bidons. Il faut aussi attendre des fabricants de machines l'invention de modalités de réglages plus commodes à mettre en œuvre, car les campagnes de réglage menées par les organismes de développement montrent qu'il y a beaucoup à faire dans ce domaine, ce qui rejoint les efforts des industriels pour fournir des formulations facilitant les dosages.

Quelles que soient les techniques, la notion de risque en matière phytosanitaire reste mal définie tant chez les utilisateurs directs, les agriculteurs, que chez les prescripteurs ; les uns et les autres ont encore trop souvent tendance à adopter des attitudes d'assurance maximum et à vouloir, ensuite, les justifier. Aussi, parmi les améliorations dans l'usage des produits phytosanitaires, on devrait mieux utiliser les connaissances déjà acquises pour déclencher des actions visant simultanément :

- à encourager les agriculteurs et leurs conseillers à mieux tenir compte des caractéristiques des matières actives 16 en interaction avec celles du milieu. Par exemple, à efficacité agronomique équivalente, privilégier de manière systématique les produits les moins toxiques parmi ceux qui sont autorisés, privilégier les produits à faibles constantes de solubilité et/ou d'échange s'il y a risque de ruissellement, incorporer en présemis les produits, chaque fois que possible, ce qui en limite le ruissellement, en particulier sur les fortes pentes $17 \ldots$

- à établir les risques de pertes au champ, en particulier du fait du climat et les localiser dans l'espace. Ainsi, le volume des pluies, l'intervalle entre le traitement et la première pluie qui le suit, l'intensité de cette dernière sont, en interaction
12 Directive 91/414/CEE, article $2, B 13$; en gras, souligné par moi-même.

13 Notre culture française ne fait guère de place aux idées de vigilance et de prévention, chacun ayant tendance à jouer sa " carte personnelle ".

14 Dans le cadre du CORPEN des documents sur cette question ont été élaborés qui insistent sur les précautions à prendre pour les manipulations à la ferme. Indiquons aussi l'existence de " Phyto-Mieux ". Cette opération lancée à l'initiative de la profession s'est surtout orientée, au moins pour l'instant, sur le thème du réglage des pulvérisateurs et des manipulations à la ferme, estimant qu'il s'agit d'un moyen commode pour atteindre les agriculteurs et, si possible, entamer un processus de vulgarisation plus ambitieux. II faut d'ailleurs remarquer que le rinçage d'une cuve effectué n'importe comment représente effectivement un risque de pollution supérieur à celui du ruissellement sur une parcelle de taille moyenne.

15 Cependant la surcharge de l'appareil qui en résulte peut se révéler nuisible dans certaines circonstances de sols humides !
16 Des auteurs ont proposé des indices pour caractériser les risques liés aux matières actives. Par exemple, pour les eaux souterraines l'indice GUS = $\log (\mathrm{DT} 50)(4-\log (\mathrm{KOC}))$. Les matières actives pour lesquelles l'indice est inférieur à 2,8 présentent peu de risques, $\mathrm{cf}$. la référence de l'article de la note 18. Citons aussi les estimations proposées par la méthode SIRIS (Système d'Intégration des Risques par Interaction de Scores) : on se reportera à " Classement des substances actives phytosanitaires en vue de la surveillance de la qualité des eaux. Fonctionnement de la méthode et recommandations pour une application régionale ". Comité de liaison "Eaux-Produits antiparasitaires ", février 1995.

17 Cf. entre autres : Hayo M.G. van der Werf, 1997. Evaluer l'impact des pesticides sur l'environnement. Le Courrier de l'environnement de I'INRA. $\mathrm{n}^{\circ} 31$, traduction d'un article paru en anglais en 1996. Voir aussi les travaux réalisés à l'ITCF : Compte Rendu du programme ACTA-MRT 93/01-6, La Jaillère. 
avec les types de sol (sols battants ou imperméables ou qui gardent une très grande " infiltrabilité ", par exemple), des facteurs très importants de risque de pertes, principalement par ruissellement. Il faut aussi connaître, selon le climat et le type de sol, la probabilité d'avoir des pluies drainantes et donc les risques de lessivage de produits phytosanitaires en profondeur vers les eaux souterraines.

Ces conseils qui présentent beaucoup d'analogies avec ceux que l'on peut donner pour l'azote montrent aussi que selon les milieux et les pratiques culturales les risques ne sont pas identiques pour les ressources en eaux, superficielles ou souterraines. On perçoit aussi que les contraintes de calendrier cultural et d'organisation du travail, elles-mêmes liées aux systèmes de culture de chaque exploitation agricole, rendront plus ou moins facile le respect de ces conseils. Par exemple, s'il doit semer une culture de printemps après une interculture d'hiver, introduite pour réduire les risques de pertes de nitrates, l'agriculteur sera souvent pressé et ne pourra, par exemple, pour réduire les risques de ruissellement, attendre que le climat soit franchement installé " au beau ". Pour ces différentes raisons, si l'on veut des conseils efficaces, ceux-ci doivent être “ ciblés ", c'est-à-dire adaptés aux situations concrètes des agriculteurs comme Ferti-Mieux essaie de le faire.

On doit noter ici les efforts que fait le CORPEN en matière de formation et d'information ${ }^{18}$, mais il manque encore beaucoup de connaissances, par exemple sur les produits issus des transformations dans le milieu, mais tout autant pour expliquer les différences de " fuites " que l'on constate dans les dispositifs expérimentaux.

\subsection{La conception des itinéraires techniques et des sys- tèmes de culture}

La notion d'itinéraire technique repose sur le fait que les différentes techniques interagissent entre elles. Par exemple, il y a déjà plus de dix ans que Meynard 19 a montré pour le blé que si l'on visait des niveaux élevés de rendement, non seulement il fallait apporter plus d'azote mais encore multiplier les traitements phytosanitaires et systématiser les apports de régulateur de croissance et, bien sûr, contrôler les mauvaises herbes par les traitements herbicides.

La mise au point de ces itinéraires techniques se complique du fait des interactions qui existent également entre les techniques culturales et les différentes variétés de chaque espèce cultivée, variétés qui se renouvellent rapidement grâce aux efforts des sélectionneurs. II n'y a donc pas, indépendamment des aléas climatiques qui exigent aussi des adaptations annuelles, une "lutte intégrée " qui pourrait être codifiée une fois pour toutes, même pour une seule espèce cultivée !

La mise au point des itinéraires techniques ne peut donc plus relever d'essais classiques, beaucoup trop réducteurs par rapport à la variabilité des pratiques agricoles possibles. Par ailleurs, on ne peut se contenter de l'expérience des agriculteurs et de leurs conseillers pour deux raisons. D'une part, la valorisation de l'expérience des agriculteurs est difficile 20 , l'interprétation des résultats culturaux étant délicate puisqu'il faut comprendre comment les conditions de l'année, son climat mais aussi les états de la terre en surface, etc., ont joué sur les rendements, et sur les risques de pollution. D'autre part, cette expérience ne permet guère, même si elle est très utile à connaître, d'anticiper, compte tenu des évolutions rapides des techniques et des variétés. On doit évoquer, à cet égard, les progrès importants obtenus en matière de lutte biologique, en culture sous serre comme au champ. Dans ce dernier cas, on connaît les résultats très encourageants de la lutte contre la pyrale du maïs avec le trichogramme 21 .

Il faut donc coupler des travaux de modélisation aux enquêtes culturales. C'est ainsi que l'un des rôles de la recherche est de mettre au point des modèles de fonctionnement du champ cultivé pour pouvoir simuler différentes combinaisons de techniques et de caractéristiques variétales, afin d'orienter les choix de lutte intégrée. Les progrès dans ce domaine sont réels et très encourageants pour l'avenir.

Mais une culture ne doit pas être considérée isolément de celles qui l'ont précédée et de celles qui la suivront ; elle est insérée dans un système de culture, nouvelle source de complexité. Voici deux exemples. Le premier concerne le travail du sol effectué sur la culture précédente. Selon qu'il aura été travaillé ou non, la position dans le sol des graines que les mauvaises herbes auront pu produire les années précédentes seront différentes et donc aussi les risques de germination, ce qui devrait, dans une optique de lutte intégrée, modifier les modalités de lutte ; de même, lorsqu'il n'y a pas eu de travail du sol, on est souvent conduit à un surdosage du fait de la présence des résidus végétaux à la surface du sol. Le second exemple a trait à la présence d'une interculture (par exemple pour réduire les risques de pollution par les nitrates). Celle-ci, selon sa nature, modifiera les risques de maladies pour la culture suivante ainsi que la manière de conduire la lutte contre les adventices.

Enfin, on doit tenir compte d'un autre aspect des successions de cultures : les dates d'implantation d'une culture dépendent plus ou moins étroitement des dates de récolte de la précédente. Cet effet a de grosses conséquences sur les conditions écologiques précises au moment d'effectuer les traitements et donc souvent sur le choix des molécules ou des formulations de produits phytosanitaires. De ce point de vue la multiplicité des molécules disponibles peut être un avantage même si elle complique la décision.

\subsection{Le rôle de l'amélioration variétale}

L'amélioration variétale est l'un des leviers puissants pour augmenter l'efficacité des méthodes de lutte contre les ennemis des cultures par l'accroissement des résistances des plantes cultivées. On oublie trop les immenses avancées obtenues dans ce domaine par les méthodes classiques de sélection. On peut estimer qu'environ la moitié des progrès de l'agriculture depuis un demi-siècle en matière de production à l'hectare a pour origine les améliorations génétiques. Cependant, face aux nouvelles perspectives qu'ouvrent les avancées récentes en matière d'organismes génétiquement modifiés (OGM), pour intéressantes qu'elles apparaissent, il faudra attendre encore quelques années pour être sûr que les performances promises seront durables et que ne se multiplieront pas des phénomènes de résistance de la part des
18 Numéro de L'Echo des nitrates et des phytos ( $\mathrm{N}^{\circ} 115$. Spécial Salon de l'Agriculture 1998) présente de manière succincte les principales brochures et plaquettes publices.

19 Meynard J.M.. 1985. Construction d'itinéraires techniques pour la conduite du blé d'hiver. Thèse Docteur Ingénieur INA PG, 297 p.
${ }^{20}$ Sans compter que les enquêtes culturales sont encore trop souvent insuffisamment renseignées et qu'elles restent de toute façon lourdes à gérer.

${ }^{21}$ Le journal Le Monde a fait écho à ces travaux le 2 mars 1998. 
populations d'ennemis des cultures. Il ne faut pas oublier non plus les voies alternatives comme celles déjà évoquées pour la pyrale du maïs.

\subsection{L'aménagement de l'espace}

Il est intéressant de distinguer deux aspects. Le premier vise à diminuer la " pression parasitaire " et la pression de sélection et donc l'apparition d'écotypes ayant des résistances en évitant de localiser des cultures aux parasites communs dans une même zone de l'espace ou en faisant se succéder trop fréquemment ces cultures sur les mêmes parcelles. Le second aspect vise à réduire les risques de pertes de matière active vers les eaux en jouant sur des dispositifs de lutte.

Les effets de l'organisation des cultures dans l'espace, ainsi que les aménagements possibles de cet espace sont certainement le domaine où la recherche est le plus en retard et où les difficultés sont les plus grandes du fait de la nécessité d'une action collective.

On parle beaucoup aujourd'hui des " bandes enherbées ", retrouvant par là les travaux menés il y a plus de vingt ans dans différents pays à propos de l'érosion. On a ainsi montré que la circulation des eaux de surface dans un bassin versant suivait un certain nombre de règles et qu'en installant des zones enherbées à des endroits précis il était possible de réduire substantiellement les risques d'érosion et les pertes en terre.

Concernant les produits phytosanitaires, différentes expérimentations ont porté sur les effets de bandes enherbées, placées sur les pentes en aval des parcelles. On a ainsi pu vérifier, à nouveau, leur efficacité pour réduire le ruissellement, mais aussi leurs effets spécifiques pour les produits phytosanitaires. La concentration de ceux-ci dans les eaux qui s'écoulent hors des bandes enherbées est fortement réduite dans les conditions des expérimentations et les quantités fixées dans la bande elle-même sont assez rapidement dégradées, il ne semble donc pas y avoir d'accumulation, ce qui est très important 22 . Néanmoins, il faut souligner que ces résultats sont liés aux conditions expérimentales qui se caractérisent, entre autres, par des parcelles de longueur courte. Le CORPEN 23 insiste, en outre, sur la localisation de ces bandes, sur la question de leur entretien et sur la nécessité d'éviter les " courts-circuits ", tels que le creusement d'une rigole en travers de la bande au moment de son implantation ...

\section{VI — LES MODALITÉS D'UNE ACTION COL- LECTIVE EN VUE DE LA PRÉVENTION}

Les différents points qui viennent d'être examinés, même s'ils ne sont pas exhaustifs, renvoient clairement à l'expérience de Ferti-Mieux, qu'il s'agisse d'élaborer ou d'adapter des références pour rendre utilisables les connaissances produites par la recherche ou d'élaborer et conduire une politique de conseil.

A côté des actions de formation déjà évoquées, il faut insister sur la nécessité d'élaborer des diagnostics collectifs et
" spatialisés ". A cet égard, le CORPEN a fait une proposition de démarche de diagnostic ${ }^{24}$, mais il existe peu d'outils et c'est un obstacle dans la situation actuelle ${ }^{25}$. Citons cependant la méthode SIRIS (cf. note 16) adoptée au niveau national pour dresser les listes de produits dans le cadre de la surveillance de la qualité des eaux. Cette dernière, malgré les questions que pose toujours l'agrégation des variables dans les méthodes de score et le fait qu'elle ne permet pas le calcul d'un risque absolu, a le mérite de permettre à des experts, qui se mettent d'accord, de comparer la " dangerosité " de matières actives dans une région, compte tenu de leur emploi réel (nombre d'hectares traités par matière active et doses pratiquées).

Un tel outil et les quelques autres qui existent, même s'ils doivent être améliorés, devraient être systématiquement utilisés et un cadre comme celui de Ferti-Mieux serait assez adéquat. En effet, Ferti-Mieux présente deux caractéristiques importantes pour un tel usage : d'une part les agriculteurs sont volontaires pour entamer une démarche de modification de leurs pratiques à risque et, d'autre part, chaque opération réunit tous les acteurs concernés par la qualité de l'eau (agriculteurs, prescripteurs, institutions publiques et administrations, syndicats des eaux...). Ces deux conditions sont indispensables pour mettre en œuvre des méthodes qui exigent un accord préalable des experts entre eux et qui imposent des décisions collectives entre les agriculteurs, et pour organiser le stockage et la capitalisation des informations sur les pratiques.

Le volontariat est même la seule démarche possible dans la mesure où il s'agit d'aller au-delà des prescriptions des " étiquettes ". C'est pourquoi les conseils doivent (et peuvent) être prodigués avec la volonté pédagogique d'augmenter progressivement les exigences au cours des années successives et d'instaurer des évaluations périodiques des modifications de pratiques avec une méthodologie spécifique pour le faire, comme à Ferti-Mieux. En effet, cette opération, véritable expérimentation sociale, se caractérise par ses exigences de rigueur, elles-mêmes garanties par le fonctionnement de son Comité Scientifique et Technique, qui instruit, après enquête sur le terrain, les dossiers destinés à l'attribution du Label Ferti-Mieux par son Comité de Pilotage 26.

Mais il ne peut y avoir d'engagement volontaire des agriculteurs que si le contrat entre les partenaires est clair et donc si l'on ne " profite" pas de la collecte d'informations ainsi faite pour, ensuite, faire insidieusement monter les exigences réglementaires. Le partenariat volontaire suppose le respect de ce principe.

\section{VII — CONCLUSION : LA NÉCESSITÉ DE LA PRÉVENTION}

En conclusion, il existe des moyens de prévention des pollutions diffuses par les produits phytosanitaires, même si de nombreuses connaissances font encore défaut. A cet égard, il faut encourager le développement des travaux de recherche ${ }^{27}$.
22 Ces résultats concernent l'ensemble des matières actives testées à I'TTCF : Real B., 1997. Etude de l'efficacité de dispositifs enherbés. ITCF-Agence de l'Eau Loire-Bretagne.

23 CORPEN, 1997. Produits phytosanitaires et dispositifs enherbés. Etat des connaissances et propositions de mise en cuvre.

24 CORPEN, 1996. Qualité des eaux et produits phytosanitaires. Proposition pour une démarche de diagnostic.

25 Cf, les recensions faites par Van der Werf (1996), op. cit., et par l'ACTA. cf. note 27
26 Evoquer Ferti-Mieux ne veut pas dire qu'il n'y a pas d'autres actions destinées à promouvoir des conseils et qu'elles ne sont pas utiles. Mais, différant dans leur esprit, elles ne comportent pas de contrôle des évolutions de pratiques et n'engagent donc pas de la même manière les agriculteurs et les acteurs de l'eau.

27 Citons les travaux de l'ACTA, 1997. Elaboration d'un outil d'évaluation des risques liés à l'utilisation des produits phytopharmaceutiques. Etude préliminaire à un projet de recherche (Dossier $n^{\circ} 96 / 12$ ). On doit aussi, bien évidemment, faire écho aux travaux de I'INRA qui, par sa réforme récente, a décidé d'accentuer son effort de recherche dans ce domaine. 
La voie réglementaire, déjà très exigeante, qui régit l'usage de ces produits du fait de leur caractère le plus souvent non naturel, a des limites parce que les conditions d'emploi, individuelles et collectives dans une région, interferent sur les risques pour l'environnement. Il faut donc aller au-delà des prescriptions obligatoires, c'est l'intérêt de tous à l'avenir. Il me semble en effet illusoire de penser que des actions curatives et une simple protection de captages soient suffisantes et économiquement acceptables. Ce sont des nappes souterraines et des bassins versants pour les eaux de surface qu'il faut protéger. Cela suppose donc des actions qui regroupent tous les partenaires concernés pour traiter simultanément le diagnostic des problèmes et la recherche des solutions, et pour cela il ne faut pas tout attendre des pouvoirs réglementaires ; chacun doit se sentir gardien du patrimoine.

Pour cela, l'exemple de Ferti-Mieux, opération consacrée à la prévention des risques de pollution diffuse par les nitrates, apporte de nombreux enseignements et ses principes, volontariat des agriculteurs et obligation d'une évaluation des modifications de pratiques, sont adaptés à la situation spécifique des produits phytosanitaires.

Mais si l'on souhaite véritablement mener pour les produits phytosanitaires des opérations s'inspirant de FertiMieux, il faut simultanément accepter ses exigences de rigueur et clarifier le " contrat " avec la société pour l'emploi des produits phytosanitaires, et ne pas " piéger" les volontaires. L'esprit à instaurer est celui d'une perception positive de l'environnement et de ses contraintes, pour bâtir une agriculture plus respectueuse des consommateurs et de l'environnement, d'une part, d'une coopération entre tous les usagers de l'eau pour trouver des solutions qui tout en préservant la qualité de l'eau tiennent compte des contraintes économiques, d'autre part. Et cela ne saurait s'obtenir par la seule voie de la réglementation. 